\title{
Training workshop on Mycobacterium whole- genome sequence data analysis
}

\author{
Yonas Kassahun Hirutu $^{1 凶}$, Mesert D Bayeleygne ${ }^{2}$, Adey F Desta ${ }^{3}$, Tewodros Tariku ${ }^{1}$, Markos Abebe ${ }^{1}$ \\ 'Armauer Hansen Research Institute (AHRI), Ethiopia \\ ${ }^{2}$ Ethiopian Biotechnology Institute (EBTI), Ethiopia \\ ${ }^{3}$ Addis Ababa University (AAU), Ethiopia \\ Competing interests: YKH none; MDB none; AFD none; TT none; MA none
}

\section{Abstract}

Basic bioinformatics training workshop conducted at Armauer Hansen Research Institute (AHRI), Addis Ababa, Ethiopia. This report describes a bioinformatics training initiative started at AHRI aiming to support life science researchers and postgraduates in handling next-generation sequence data.

\section{Introduction}

Institutional initiatives strengthening capacity at Armauer Hansen Research Institute (AHRI) focuses on building a bioinformatics training centre, a nextgeneration sequencing (NGS) facility and a computing platform to support researchers and postgraduates in Ethiopia. The faculties benefit both ongoing and new project initiatives such as on pathogen evolution, virulence determinants and epidemiology of important pathogens, including $M$. tuberculosis.

The workshop aimed at delivering a practical introduction to NGS data analysis of the M. tuberculosis complex (MTBC) genome.

Every workshop day included 40 minutes presentation, three hours hands-on practical and 20 minutes discussion. The presentation topics were on next-generation sequencing technologies, examples of

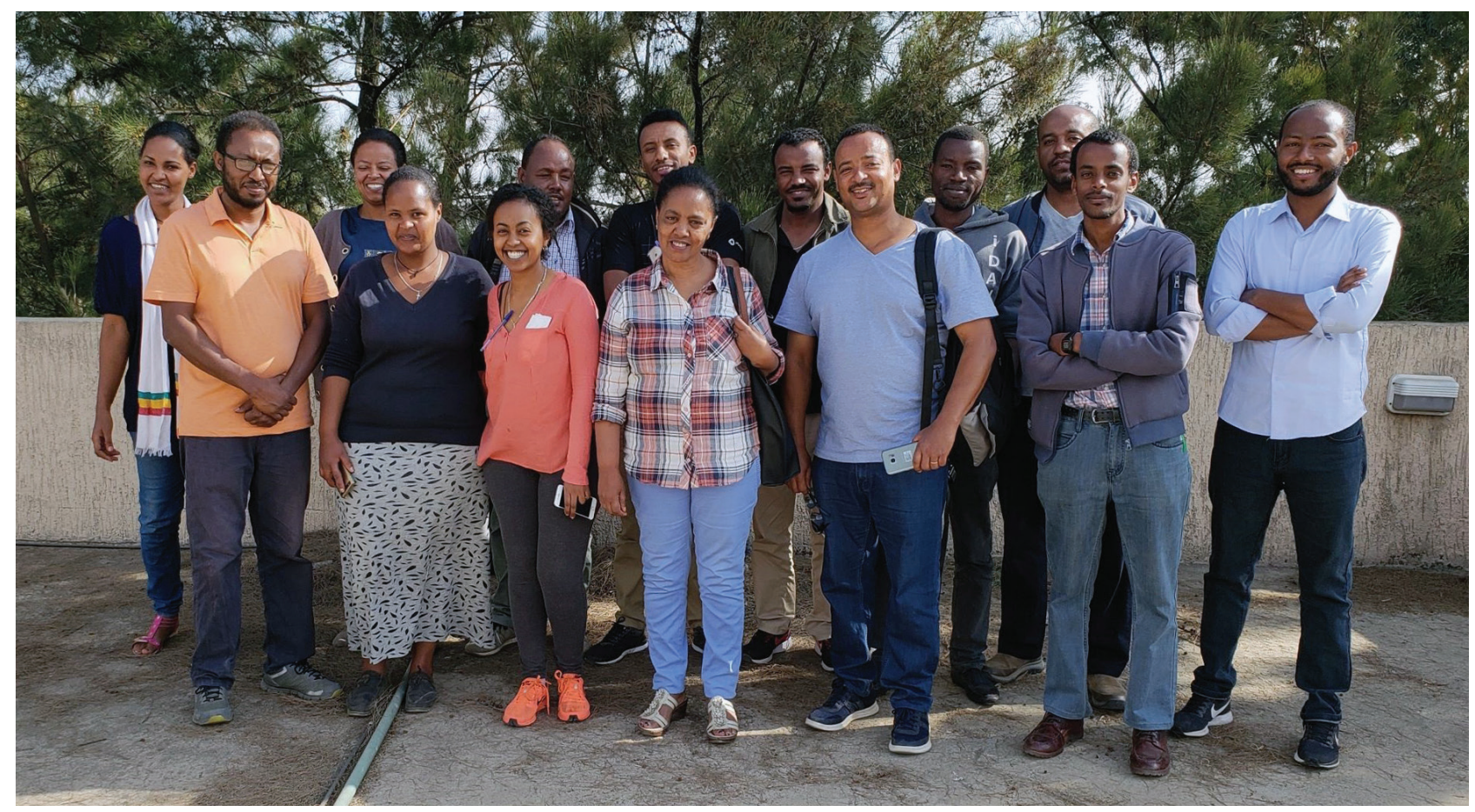

Figure 1. Participants and trainers of the Workshop

\section{Article history}

Received: 15 January 2019

Accepted: 21 September 2019

Published: 15 October 2019

(C) 2019 Hirutu et al.; the authors have retained copyright and granted the Journal right of first publication; the work has been simultaneously released under a Creative Commons Attribution Licence, which allows others to share the work, while acknowledging the original authorship and initial publication in this Journal. The full licence notice is available at http://journal.embnet.org. 
sequence data file formats and stepwise description of each NGS data analysis bioinformatics workflow.

The workshop was held at the Armauer Hansen Research Institute (AHRI), Bioinformatics unit, 24-28 November 2018. There were 12 participants from health and biotechnology research institutes in Ethiopia (Figure $1)$.

\section{Implementation of the workshop}

The workshop started with a lecture on Linux and command-line tools. AHRI's computing room with Bio-Linux workstations was used for demonstrations and practical sessions. Each workstation was assigned for two participants during the workshop. The first-day activity was focused on giving adequate practice time and guidance on command line environment.

Illumina paired-end reads of three Ethiopian $M$. tuberculosis complex (MTBC) strains were selected for the practical sessions. The strains were subsets of fastq files stored at AHRI for which NGS runs were outsourced as part of previous studies in the institute. Nine MTBC genome sequences were downloaded from $\mathrm{NCBI}^{1} . M$. tuberculosis H37Rv (NC_000962) was used as a reference genome for mapping, variant calling and annotation of MTBC strains.

The practical workflow analysis included quality control of reads with the FastQC tool (Andrews, 2010). Duplicates were removed using a custom Python script. The reads were aligned by Burrows-Wheeler Alignment Tool (BWA) with default parameters for each sample (Li and Durbin, 2009). The aligned results were piped to SAMtools for the conversion of BWA output format to BAM format (Li et al., 2009). Finally, a consensus variant file (VCF) was generated with BCFtools for subsequent SNPanalysis (Danecek etal., 2011). The variantsidentified with SAMtools/ BCFtools were inspected using the Integrative Genomics Viewer (IGV) (Thorvaldsdottir et al., 2013).The reads were also analysed with the MTBseq tool which is a comprehensive pipeline for wholegenome sequence analysis of $\mathrm{M}$. tuberculosis complex isolates with a full workflow functionality implemented in Perl modules (Kohl et al., 2018).

MTBseq analysis tool is designed for MTBC NGS data for reference mapping, variant detection, variant annotation for drug resistance and comparative analysis among the samples. MTBseq tool was used to demonstrate additional approaches and summarise a consolidated view of all the steps followed during the practical workshop. MTBseq annotation outputs of amino acid changes for a known association to antibiotic resistance were used to characterise the samples. Variants information file was used to construct phylogenetic tree using FastTree (Price et al., 2010) (Figure 2).

Finally, a presentation was made on NGS analysis of metagenomics data. The workshop participants had an opportunity to reflect on their training experience and comment on different aspects. Participants' feedback highlighted the need to improve computational

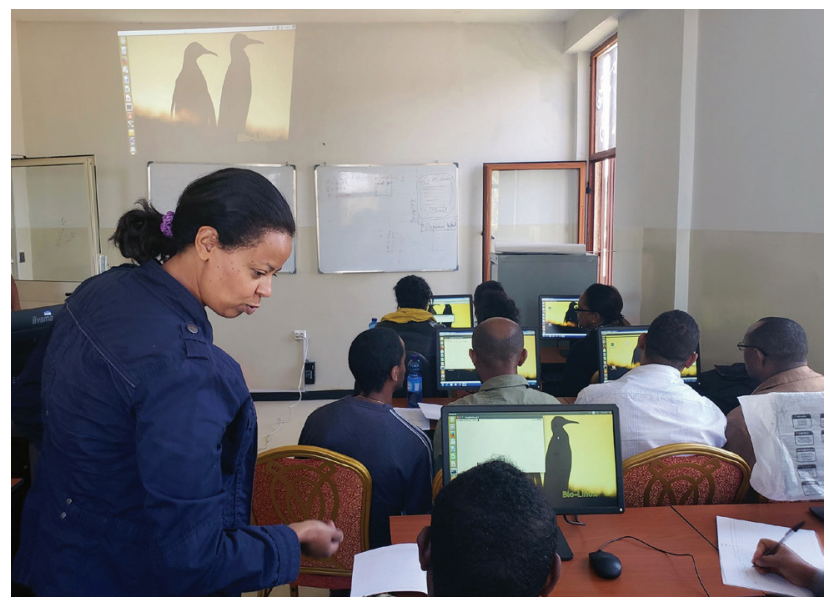

Figure 2. Workshop participants during the hands-on session.

power of the workstations and address fluctuations of internet connectivity. The participants also expressed a satisfactory level for acquiring basic skills in handling NGS data.

In conclusion, the training coordinators and leaders of the institute have appreciated the success of the workshop. The trainers acknowledged the participants for their active engagement throughout the training week.

\section{Acknowledgements}

The workshop was supported by training and research directorate at AHRI with a funding source from the Swedish International Development Cooperation Agency (SIDA). The authors would also like to acknowledge support from the African Academy of Sciences (AESA) through Tuberculosis Genetics Network in Africa (TBGENAfrica) research project at AHRI.

\section{References}

1. Andrews S. (2010)FastQC: a quality control tool for high throughput sequence data. http://www.bioinformatics.babraham. ac.uk/projects/fastqc (accessed 20 October 2018)

2. Li H and Durbin R. (2009) Fast and accurate short read alignment with Burrows-Wheeler transform.Bioinformatics 25: 1754-1760 http://dx.doi.org/10.1093/bioinformatics/btp324

3. Li H, Handsaker B, Wysoker A, Fennell T, Ruan J, Homer N, Marth G, Abecasis G, Durbin R (2009) The Sequence Alignment/ Map format and SAMtools. Bioinformatics 25: 2078-2079. http:// dx.doi.org/10.1093/bioinformatics/btp352

4. Danecek P, Auton A, Abecasis G, Albers CA, Banks E et al. (2011) The variant call format and VCFtools. Bioinformatics 27: 21562158. http://dx.doi.org/10.1093/bioinformatics/btr330

5. Thorvaldsdottir H, Robinson JT, Mesirov JP (2013) Integrative Genomics Viewer (IGV): high-performance genomics data visualization and exploration. BriefBioinform. 14: 178-192. http://dx.doi.org/10.1093/bib/bbs017.

6. Kohl TA, Utpatel C, Schleusener V, De Filippo MR, Beckert P, Cirillo DM, Niemann S. (2018) MTBseq: a comprehensive pipeline for whole genome sequence analysis of Mycobacterium tuberculosis complex isolates. PeerJ6:e5895.http://dx.doi.org/. $10.7717 /$ peerj.5895

7. Price MN, Dehal PS, Arkin AP (2010) FastTree 2 - Approximately Maximum-Likelihood Trees for Large Alignments. PLoSONE5:e9490. http://dx.doi.org/10.1371/journal. pone. 0009490 